\title{
Clinical and Demographical Characteristics of Patients with Medication Overuse Headache in Argentina and Chile: Analysis of the Latin American Section of COMOESTAS Project
}

\author{
Beatriz Shand ${ }^{1}$, Maria Teresa Goicochea ${ }^{2}$, Raul Valenzuela ${ }^{1}$, Ricardo Fadic ${ }^{1 *}$, Rigmor Jensen ${ }^{3}$, Cristina Tassorelli ${ }^{4,5}$, \\ Giuseppe Nappi ${ }^{4}$ and the COMOESTAS CONSORTIUM
}

\begin{abstract}
Background: Data on the characteristics of Medication Overuse Headache $(\mathrm{MOH})$ in Latin American (LA) are scarce. Here we report the demographic and clinical features of the $\mathrm{MOH}$ patients from Argentina and Chile enrolled in the multinational COMOESTAS project in the period 2008-2010.

Methods: The LA population was formed by $240 \mathrm{MOH}$ subjects, 110 from Chile and 130 from Argentina, consecutively attending the local headache centres. In each centre, specifically trained neurologist interviewed and confirmed the diagnosis according to the ICHD-II criteria. A detailed history was collected on an electronic patient record form.

Results: The mean patient age was 38.6 years, with a female/male ratio of 8:2. The mean time since onset of the primary headache was 21 years, whereas duration of $\mathrm{MOH}$ was 3.9 years. The primary headache was migraine without aura in $77.5 \%$ and migraine with aura in $18.8 \%$. Forty two \% of the patients self-reported emotional stress associated with the chronification of headache; $43.8 \%$ reported insomnia. The most overused medications were acute drug combinations containing ergotamine (70\%), NSAIDs (33.8\%) and triptans (5.4\%).
\end{abstract}

Conclusion: Though little described, $\mathrm{MOH}$ is present also in $\mathrm{LA}$, where it affects mostly women, in the most active decades of life. Some differences emerge as regards the demographic and clinical characteristics of $\mathrm{MOH}$ in this population as compared to Europe or Northern America. What seems more worrying about MOH in Argentina and Chile is that most patients overuse ergotamine, a drug that may cause serious adverse events when used chronically. These findings once more underscore the importance of properly diagnose and treat $\mathrm{MOH}$.

Keywords: Medication Overuse Headache $(\mathrm{MOH})$; Chronic headache; Ergotamine

\section{Background}

Medication overuse headache $(\mathrm{MOH})$ is defined by the chronification of an episodic headache in association with the excessive use of any type of symptomatic medication. It has been recognized for over 50 years [1]. It primarily affects women (3.5:1), with an average age of 40 and is usually diagnosed several years after overuse has started $[2,3]$. In the vast majority of patients, it is possible to recognize a previous primary headache, in most cases represented by

\footnotetext{
* Correspondence: rfadic@med.puc.cl

'Department of Neurology, Pontificia Universidad Católica of Chile, Santiago, Chile

Full list of author information is available at the end of the article
}

migraine $[4,5]$. Triptans and non steroid anti-inflammatory drugs (NSAIDs) are the main prescription drugs overused in Western Europe [6, 7]. Some risk factors have been proposed for $\mathrm{MOH}$ : high frequency of both pain medication use and pain episodes, use of two or more different medications, coexistence of other body pains, high levels of daily stress, low socio-economic status, psychiatric comorbidities $[1,8,9]$. European epidemiological studies have shown a prevalence of 1 to $3 \%$ [10-12], but in specialized headache centres the percentage markedly increases $[13,14]$. Precise epidemiological data about the prevalence of $\mathrm{MOH}$ in Latin 
Americaare lacking. In Chile, for instance, though the condition has been known for many years [15], there is only one publication investigating the epidemiology of chronic headache, but not focused on $\mathrm{MOH}$ [16]. In this paper we present the main demographic and clinical characteristics of $\mathrm{MOH}$ in a population of $240 \mathrm{MOH}$ sufferers living in Argentina and Chile.

\section{Methods}

We evaluated 240 patients consecutively visited in the Neurology Department of Pontificia Universidad Católica de Chile (PUC) and in the Integral Pain Center at the Fundación para la Lucha contra las Enfermedades Neurológicas Infantiles (FLENI), Argentina, from January 2008 to December 2010 by neurologists who underwent the specific training for $\mathrm{MOH}$ diagnosis developed within the COMOESTAS project $[17,18]$. Both LA institutions take care of privately insured patients. Most of the patients included in the protocol were recruited during spontaneous headache consultation at the outpatient clinics both centers $(98.3 \%)$. Very few patients were recruited while hospitalized. As patients were enrolled before the publication of ICDH-IIIbeta [19], the diagnosis of primary headaches (migraine, chronic migraine and tension-type headache) and $\mathrm{MOH}$ was made on ICHDII [20] and ICHDII-R criteria [21]. For the scopes of the present paper, diagnoses were then reviewed and confirmed retrospectively using the ICHDIII beta criteria.

Demographic data, habits, personal and family medical history were recorded, as well as $\mathrm{MOH}$ history, $\mathrm{MOH}$ characteristics and medications being overused. All the information included in this paper was obtained through a thorough anamnestic report.

The data were collected within the activities of the COMOESTAS project, which obtained the approval of the Research Ethics Committee at the Medical School of Pontificia Universidad Católica de Chile and the Ethics Committee and Biomedical Research at FLENI. Written consent was obtained for all patients.

\section{Statistical analysis}

The categorical variables were described as absolute and relative frequency. The continuous variables were described as mean and standard deviation. The level of significance was set to $\mathrm{p}<0.05$.

All of the analyses were done with the Program: SPSS Inc. Released 2008. SPSS Statistics for Windows, Version 17.0. Chicago: SPSS Inc.

\section{Results}

Of the 240 patients evaluated, 110 were enrolled in Chile and 130 in Argentina. The mean age was 38.7 years (range: $18-72$ ), $80.4 \%$ of patients were women, $75 \%$ had a technical or university educational level. The main characteristics of the study population are given in Table 1.

\section{Headache characteristics}

Migraine without aura was the most common primary headache $(77.8 \%)$ prior to $\mathrm{MOH}$. In decreasing order, we found migraine with aura (18.8\%), chronic tensiontype headache (15.9\%), and episodic tension-type headache $(8.4 \%)$. A minority of patients had chronic migraine as their primary headache. In a minority of patients two types of primary headaches coexisted. Some patients did have more than one headache type. The average age of onset of the primary headache was 17.7 years. Noteworthy, in $52.3 \%$ of subjects, headache started below the age of 16 . Headache chronification was related to self-reported stress in $42.7 \%$ of patients.

\section{Related conditions and habits}

Mood and anxiety disorder were common in our patients past medical history: depression was reported in $26.3 \%$, anxiety in $20.8 \%$, and insomnia in $43.8 \%$. Hypertension was present in $12.9 \%$. Family history was positive for depression in $32.5 \%$, for alcoholism in $17.5 \%$ and for any type of headache in $73.3 \%$. Habits findings were notable as regards the use of phosphodiesterase inhibitors beverages on a daily basis: coffee $51.7 \%$, tea $51.7 \%$, cola beverages $33.3 \%$ and mate $27.1 \%$, in most cases in association. $44.2 \%$ of patients drank a moderate amount of alcohol (less than $0.5 \mathrm{~L}$ of wine per day) and $26.3 \%$ smoked cigarettes.

\section{Medications overused}

Average length of overuse was 3.9 years (range 1-30 years). In this population, the majority of subjects overused ergotamines $(70 \%$, alone or combined with NSAIDs or caffeine), $33.9 \%$ overused exclusively NSAIDs, $6.3 \%$ combination drugs, and only $5.4 \%$ triptans alone (more than one type of triptans in some cases). The clinical characteristics of patients are given in Table 2.

\section{Use of medical resources}

In the year prior to the evaluation, $30.1 \%$ of our patients sought help at the emergency room at least once due to

Table 1 Demographic characteristics of the population

\begin{tabular}{llll}
\hline & Medium & Sd \\
\hline Age & 38.7 & & 11.9 \\
Gender (female/male) & $201 / 39$ & & \\
Married/single & $125 / 115$ & & \\
Educational level & Primary & Secondary & Technical degree \\
& 14 & 46 & 68 \\
& & & University degree \\
& & & 112 \\
\hline
\end{tabular}


Table 2 Clinical characteristics of the population

\begin{tabular}{lll}
\hline & Medium & $\mathrm{Sd}$ \\
\hline Age at onset & 17.7 & 8.5 \\
(years) & & \\
Days of drug intakes/month & 21.5 & 6.9 \\
Headaches days/month & 22.4 & 6.1 \\
Duration of overuse (years) & 3.9 & 4.4 \\
\hline
\end{tabular}

headache, with a range of 1 to 20 visits. $26.8 \%$ consulted a general physician and $37.7 \%$ went for a neurological evaluation at least once. $27.5 \%$ of participants had a head computerized tomography in the last year.

\section{Discussion}

The Latin American section of the COMOESTAS Project represents the largest group of $\mathrm{MOH}$ patients published from this region. The demographic profile that has emerged is similar to the published data from developed countries [22], though it is worth noting that both participating medical centers treat patients with private health insurance, which explains the high educational level of the group studied. Our results might therefore not be representative of the general population in our countries. It is necessary to note that Argentina and Chile are the countries with the highest level of education and income in the region; therefore caution must be used when attempting to extrapolate these results to other Latin America countries with certainly.

In agreement with other reports on $\mathrm{MOH}$ in other parts of the world, also in this series migraine is by far the most frequently reported primary headache $[15,16,23]$. The average medication overuse time in this series is significantly shorter than previously described [3-5], even though the time passed since the onset of the primary headache is similar. This finding may be related to the exclusion criteria of the COMOESTAS Project, which required naif $\mathrm{MOH}$ subjects (patients without a history of previous failure in detoxification therapy).

In the present series, ergotamine is the drug most frequently overused. This is likely due to the fact that medications containing ergotamine are both the cheapest and most readily available headache treatments in Chile and Argentina, where they are sold without prescription.

Similar to the Katsarava's studies $[8,9]$ a relevant percentage of patients in this series made a relation between chronification of pain and stressful situations. At variance, the percentage of subjects with diagnosis of depression or anxiety in our population seems lower than reported in other countries [24]. Even though this could be related to the different methods used for diagnosing depression in the different studies (here we adopted Hospital Anxiety and Depression Scale, HADS), the lower frequency of depression could be linked to the shorter duration of overuse in our series. This raises the possibility that depression and anxiety in $\mathrm{MOH}$ may be a consequence of chronic pain, rather than a risk factor.

The tendency to consult a neurologist instead of a primary physician for headaches is characteristic of our study population. Most of our patients are privately insured and tend to choose for themselves who will provide their care. This is a feature of our mixed health insurance system with some patients being served by a public system, while other have access to a private one.

\section{Conclusion}

In conclusion, though little described, $\mathrm{MOH}$ is present also in LA, where it affects mostly women, in the most active decades of life. Some differences emerge as regards the demographic and clinical characteristics of $\mathrm{MOH}$ in this LA population when compared to Europe or Northern America. What seems more worrying about $\mathrm{MOH}$ in Argentina and Chile is that most patients overuse ergotamine, a drug that has been banned from many European countries because of the high risk of toxicity associated to its chronic use. These findings once more underscore the importance of properly diagnose and treat $\mathrm{MOH}$.

\section{Abbreviations}

COMOESTAS: Continuous Monitoring of Medication Overuse Headache in Europe and Latin America; HADS: Hospital Anxiety and Depression Scale; IHS: International Headache Society; $\mathrm{MOH}$ : Medication Overuse Headache.

\section{Competing interests}

The authors declare that they have no competing interests.

\section{Author's contribution}

BS participated acquiring and analyzing data, drafting the manuscript. MTG acquiring and analyzing data, reviewing critically the manuscript. RV acquiring and analyzing data, reviewing the manuscript. RF participated designing the study, acquiring and analyzing data, drafting the manuscript. RJ, CT, GN participated designing the study and reviewing critically the manuscript. All the authors have given final approval of the version to be published.

\section{Acknowledgements}

This work has been funded by by COMOESTAS Project - EC contract number 215366 (COMOESTAS: Continuous Monitoring of Medication Overuse Headache in Europe and Latin America: Development and Standardization of an Alert and Decision Support System) FP7 - Thematic priority ICT. The work has been conducted in collaboration with the University Consortium for Adaptive Disorders and Head pain.

We wish to thank Mr. Orlando Padilla for his contribution with the statistical analysis. COMOESTAS Consortium: M. Allena, G. Sances, G. Sandrini, F. Blandini, P. Rossi (Headache Science Centre C. Mondino National Neurological Institute), L. Bendtsen (Región Hoveedstaden,GlostrupAmtssygehuset, Dinamarca), M. Lainez (Fundación de la Comunidad Valenciana para la Investigación Biomédica, la Docencia y laCooperación Internacional y para el Desarrollo del Hospital Clínico Universitario deValencia, Spain), Z. Katzarava, D. Mueller (Universitaetklinikum Essen, Germany).

\section{Author details}

'Department of Neurology, Pontificia Universidad Católica of Chile, Santiago, Chile. ${ }^{2}$ Integral Pain Centre, Fundación para la Lucha contra las Enfermedades Neurológicas Infantiles (FLENI), Buenos Aires, Argentina. ${ }^{3}$ Danish Headache Centre, Glostrup Amtssygehuset, University of Copenhagen, Copenhagen, Denmark. ${ }^{4}$ Headache Science Centre, C. Mondino National Neurological Institute, Pavia, Italy. ${ }^{5}$ Department of Brain and Behavioral Sciences, University of Pavia, Pavia, Italy. 
Received: 14 April 2015 Accepted: 1 August 2015

Published online: 18 September 2015

\section{References}

1. Munskgaard S, Jensen R (2014) Medication overuse headache. Headache 54(7):1251-1257

2. Silberstein S (2003) Chronic daily headache. Continuum: Headache Update 9:121-143

3. Zeeberg P, Olesen J, Jensen R (2005) Efficacy of multidisciplinary treatment in a tertiary referral headache centre. Cephalalgia 25(12):1159-1167

4. Diener HC, Limmroth V (2004) Medication-overuse headache: a worldwide problem. Lancet Neurol 3(8):475-483

5. Haag G, Baar H, Grotemeyer KH et al. (1999) Prophylaxis and treatment of drug-induced persistent headache. Therapy recommendation of the German Society for Migraine and Headache. Schmerz 13(1):52-57

6. Kristoffersen E, Lundqvist C (2014) Medication-overuse headache: a review (2014). J Pain Res 26:367-378

7. Ghiotto N, Sances G, Galli F et al. (2009) Medication overuse headache and applicability of the ICHD-II diagnostic criteria: 1-year follow-up study (CARE I protocol). Cephalalgia 29:233-243

8. Katsarava Z, Obermann M (2013) Medication-overuse headache. Curr Opin Neurol 26(3):276-281

9. Hagen $K$, Linde M, Steiner T et al (2012) Risk factor for medication-overuse headache: an 11 year-follow-up study. The Nord- Trondelag Health Studies. Pain 153:56-61

10. Zebenholzer K, Andree C, Lechner A, Broessner G, Lampl C, Luthringshausen G, Wuschitz A, Obmann SM, Berek K, Wöber C (2015) Prevalence, management and burden ofepisodic and chronic headaches-a cross-sectional multicentre study in eightAustrian headache centres. J Headache Pain 16:531

11. Westergaard ML, Glümer C, Hansen EH, Jensen RH (2014) Prevalence of chronic headache with and without medication overuse: associations with socioeconomic position and physical and mental health status. Pain 155:2005-2013

12. Steiner TJ, Stovner LJ, Katsarava Z, Lainez JM, Lampl C, Lantéri-Minet M, Rastenyte D, Ruiz de la Torre E, Tassorelli C, Barré J, Andrée C (2014) The impact ofheadache in Europe: principal results of the Eurolight project. J Headache Pain 15:31

13. Vinding GR, Zeeberg P, Lyngberg A et al. (2007) The burden of headache in a patient population from a specialized headache centre. Cephalalgia 27(3):263-270

14. Rapoport A, Stang P, Gutterman DL, Cady R, Markley H, Weeks R et al. (1996) Analgesic rebound headache in clinical practice: data from a physician survey. Headache 36(1):14-19

15. Nogales-Gaete J (1992) Jaqueca por dependencia de tartrato de ergotamina. Rev Med Chile 120(7):797-799

16. Castillo J, Muñoz P, Guitera V et al. (1999) Epidemiology of chronic daily headache in general population. Headache 39(3):190-196

17. Bendtsen L, Munksgaard S, Tassorelli C et al. (2014) Disability, anxiety and depression associated with medication-overuse headache can be considerably reduced by detoxification and prophylactic treatment. Results from a multicentre, multinational study (COMOESTAS project). Cephalalgia 34(6):426-433

18. Tassorelli $C$, Jensen $R$, Allena $M$ et al. (2014) A consensus protocol for the management of medication-overuse headache: Evaluation in a multicentric, multinational study. Cephalalgia 34(9):645-655

19. Headache Classification Committee of the International Headache Society (IHS) (2013) The International Classification of Headache Disorders, 3rd edition (beta version). Cephalalgia 33:629-808

20. Headache Classification Committee of International Headache Society (2004) The International Classification of Headache Disorders. 2nd ed. Cephalalgia 24(Suppl 1):1-160

21. Headache Classification Committee (2006) New appendix criteria open for a broader concept of chronic migraine. Cephalalgia 26:742-746

22. Jonsson P, Linde M, Hensing G, Hedenrud T (2012) Sociodemographic differences inmedication use, health-care contacts and sickness absence among individuals with medication-overuse headache. J Headache Pain 13(4):281-290
23. Diener HC, Dahlöf CGH (1999) Headache associated with chronic use of substances. In: Oleson J, Tfelt-Hansen P, Welch KMA (eds) The headaches, 2nd edn. Lippincott, Philadelphia, pp 871-878

24. Atasoy HT, Atasoy N, Unal AE, Emre U, Sumer M (2005) Psychiatric comorbidity inmedication overuse headache patients with pre-existing headache type of episodic tension-type headache. Eur J Pain 9(3):285-291

\section{Submit your manuscript to a SpringerOpen ${ }^{\odot}$ journal and benefit from:}

- Convenient online submission

- Rigorous peer review

- Immediate publication on acceptance

- Open access: articles freely available online

- High visibility within the field

- Retaining the copyright to your article

Submit your next manuscript at $>$ springeropen.com 